\title{
Research Paper \\ The Role of Self-compassion in Predicting Loneliness and Self-efficacy in the Elderly
}

\author{
*Mehdi Ghezelseflo ${ }^{1}$ (ㅇ, Mozhgan Mirza ${ }^{1}$
}

1. Department of Psychology, Faculty of Humanities and Physical Education, Gonbad Kavous University, Gonbad Kavous, Iran.

\begin{tabular}{|c|c|}
\hline $\begin{array}{l}\text { Use vour device to san } \\
\text { and read the article online }\end{array}$ & \\
\hline 口ificia & $\begin{array}{l}\text { dtration: Ghezelseflo M, Mirza M. [The Role of Self-compassion in Predicting Loneliness and Self-efficacy in the Elderly } \\
\text { (Persian)]. Iranian Journal of Ageing. 2020; 15(2):212-223. https://doi.org/10.32598/sija.13.10.630 }\end{array}$ \\
\hline DFtrits & dol'https://doi.org/10.32598/sija.13.10.630 \\
\hline
\end{tabular}

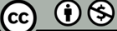

Received: 13 Dec 2017 Accepted: 09 Jul 2019 Available Online: 01 July 2020

Key words: Self-compassion, Loneliness, Self-efficacy, Elderly

\section{A B S TRACT}

Objectives Giving the growing population of the elderly, it is important to pay attention to their psychological health. The purpose of this study was to examine the role of self-compassion in predicting loneliness and self-efficacy in the elderly.

Methods \& Materials This is a descriptive correlational study. Study population consisted of all elderly residents of Gonbad Kavous County in Iran. Of these, 120 (70 women and 50 men) were selected using a convenience sampling technique. Data collection instruments were University of California, Los Angeles (UCLA) Loneliness Scale of Russell et al. (1966), General Self-efficacy Scale of Scherer et al. (1982) and SelfCompassion Scale of Neff (2003). Data analysis was performed by using Pearson correlation coefficient and multiple regression analysis.

Results Loneliness had a significant negative relationship with self-compassion dimensions of self-kindness, common humanity, mindfulness and its overall score, and a significant positive relationship with self-judgment, isolation and over-identification. Moreover, self-efficacy had a significant positive relationship with self-kindness, common humanity, mindfulness and overall self-compassion score, and a negative significant relationship with self-judgment, isolation and over-identification ( $\mathrm{P} \leq 0.001)$. Self-kindness, self-judgment, common humanity, isolation, mindfulness and overall self-compassion score can predict $43 \%$ of loneliness variance and self-kindness, self-judgment, mindfulness, isolation and overall self-compassion score can predict $38 \%$ of self-efficacy variance.

Conclusion Self-compassion have an effective role in reducing loneliness and increasing self-efficacy in the elderly.

\section{Extended Abstract}

\section{Introduction}

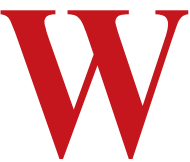

ith the onset of old age, people gradually lose some of their physiological, psychological and social functions. Deprivation of social activities increases the feeling of loneliness in them [1]. One of the components that can play an effective role in reducing their loneliness is the self-compassion. Self-compassion is a structure that consists of three components: a. self-kindness rather than being self-critical; b. common humanity rather than seeing them as separating and isolating; and c. mindfulness rather than over-identifying negative thoughts and feelings; [2].

\section{* Corresponding Author:}

Mehdi Ghezelseflo, PhD.

Address: Department of Psychology, Faculty of Humanities and Physical Education, Gonbad Kavous University, Gonbad Kavous, Iran.

Tel: +98 (912) 7929287

E-mail: me.ghezelseflo@gmail.com 
Studies have shown that self-compassion is positively correlated with many positive psychological constructs [3]. One of these structures is self-efficacy [2]. Neff showed that self-compassion has a significant positive correlation with competence and autonomy [3].

\section{Methods \& Materials}

This is a descriptive correlational study. Study population consisted of all elderly residents of Gonbad Kavous County aged 60-80 years in 2017. A sample size of 120 was determined by Cochran formula, and samples were selected by using a convenience sampling method (70 women and 50 men) among those were in urban parks. Inclusion criteria were age over 60 years, ability to complete questionnaires, a minimum elementary education for reading and writing, and willingness to participate in the study. Those who were unwillingness were excluded from the study. After obtaining ethical approval from Gonbad Kavous University, participants completed: a. University of California, Los Angeles (UCLA) Loneliness Scale developed by Russell et al. [4] in 1978 which has 20 items (11 negatively worded and 9 positively worded); b. Self-Compassion Scale (SCS) developed by Neff; [5] which has 26 items and 6 subscales of Self-kindness (5 items), self-judgment (5 items), Common humanity (4 items), isolation(4 items), Mindfulness (4 items), and over-identification (4 items); c. General SelfEfficacy Scale (GSE) developed by Sherer et al. [6] which has 17 items rated on a 5-point Likert scale ( from 1: Totally agree to 5: Totally disagree).

\section{Results}

The Mean \pm SD age of women was $64.22 \pm 4.42$ years and the mean age of men was $66.25 \pm 3.52$ years. The results presented in Table 1 showed a negative significant relationship of loneliness with SCS dimensions of self-kindness ( $\mathrm{r}=-0.60, \mathrm{P}=0.000)$, Common humanity $(\mathrm{r}=-0.58, \mathrm{P}=0.000)$, Mindfulness $(\mathrm{r}=-0.65, \mathrm{P}=0.000)$ and its overall score $(\mathrm{r}=$ $0.42, \mathrm{P}=0.000$ ), while had a positive significant relationship with SCS dimensions of self-judgment $(\mathrm{r}=0.59, \mathrm{P}=0.000)$, isolation $(\mathrm{r}=0.56, \mathrm{P}<0.05)$, and over-identification $(\mathrm{r}=0.53$, $\mathrm{P}<0.05$ ). Moreover, the results showed the positive significant relationship of self-efficacy with self-kindness $(r=0.47$, $\mathrm{P}<0.05$ ), common humanity $(\mathrm{r}=0.57, \mathrm{P}<0.05)$, mindfulness $(\mathrm{r}=0.62, \mathrm{P}<0.05)$ and its overall score $(\mathrm{r}=0.46, \mathrm{P}=0.000)$; also its negative significant relationship with self-judgment $(\mathrm{r}=-0.63, \mathrm{P}=0.000)$, isolation $(\mathrm{r}=-0.53, \mathrm{P}<0.05)$, and overidentification $(\mathrm{r}=-0.52, \mathrm{P}<0.05)$. The linear regression of the predictor variables in explaining loneliness was significant with $\mathrm{F}_{2,117}=31.37, \mathrm{P}<0.05$, and adjusted $\mathrm{R}=0.43$. That is, about $43 \%$ of the variance of loneliness can be explained by the predictor variables except the over-identification variable. The linear regression of the predictor variables in explaining self-efficacy was significant with $\mathrm{F}_{2,117}=25.07$, $\mathrm{P}<0.05$, and adjusted $\mathrm{R}=0.38$ indicating that $18 \%$ of the selfefficacy variance can be explained by the predictor variables except common humanity and over-identification variables.

Table 1. Correlation coefficients between variables and summary of linear regression analysis

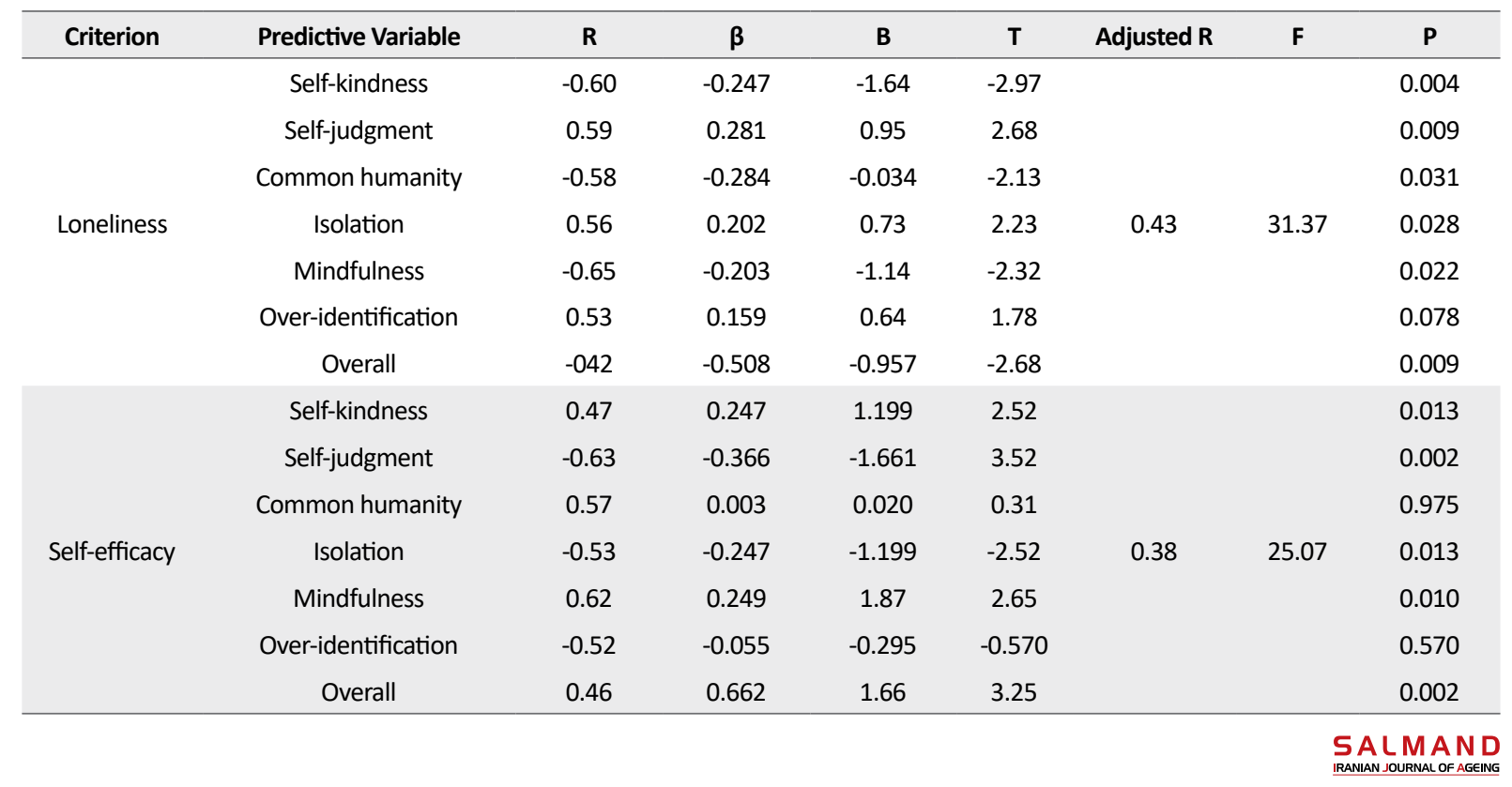




\section{Conclusion}

Loneliness in older people had a negative significant relationship with self-compassion dimensions of self-kindness, common humanity, mindfulness, and in overall. This means that by being kind toward self, perceiving self's experiences as part of the larger human experience, and holding painful thoughts and feelings in balanced awareness, they feel less lonely. Their loneliness had also a positive significant relationship with self-compassion dimensions of self-judgment, isolation, and over-identification. Moreover, self-efficacy in the elderly people had a positive significant relationship with self-kindness, common humanity, mindfulness, and in overall, while had a negative significant relationship with self-judgment, isolation, and over-identification. Furthermore, self-compassion was able to predict loneliness and self-efficacy in the elderly. It can be concluded that high self-compassion negatively predicts feelings of loneliness and positively predicts self-efficacy in older adults. In other words, with improved self-compassion, older people will have less loneliness and higher self-efficacy.

\section{Ethical Considerations}

\section{Compliance with ethical guidelines}

All ethical principles are considered in this article. The participantswere informed about the purpose of the research andits implementation stages; they were also assured about theconfidentiality of their information; moreover, they were freeto leave the study whenever they wished, and if desired, the research results would be available to them.

\section{Funding}

This research did not receive any grant from funding agencies in the public, commercial, or non-profit sectors.

\section{Authors' contributions}

Conceptualization, Investigation, Resources, Writing -original draft preparation, Funding acquisition: Both authors; Methodology, Writing-review \& editing, Formal analysis, Data curation, Supervision: Mehdi Ghezelseflo.

\section{Conflicts of interest}

The authors declared no conflict of interest. 


\title{
نقش خوددلسوزى در بيشبينى احساس تنهايى وخودكار آمدى سالمندان
}

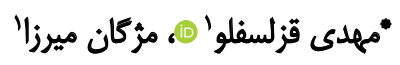

ا. كروه روانشناسى، دانشكده علوم انسانى و تربيتى، دانشَّاه كنبد كاووس، كنبد كاووس، يران.

\section{حكيد}

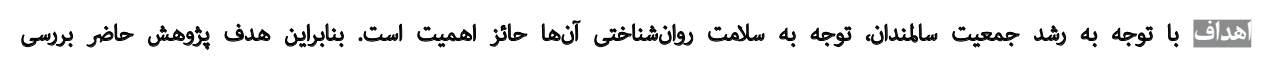

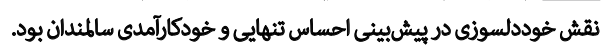

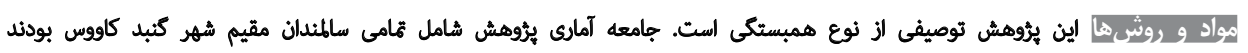

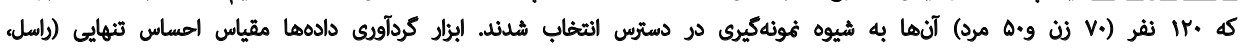

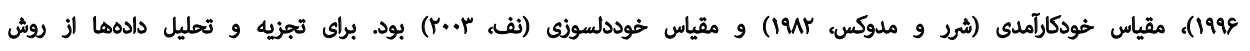

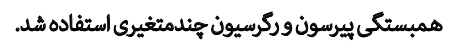

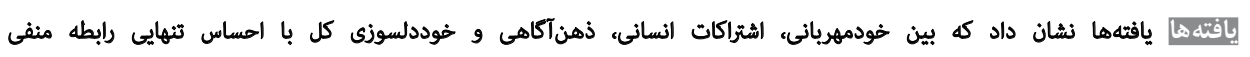

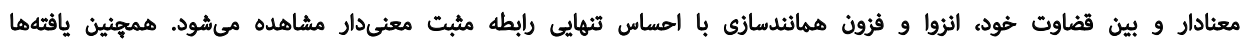

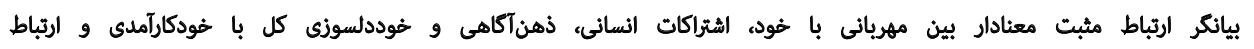

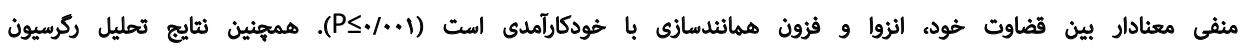

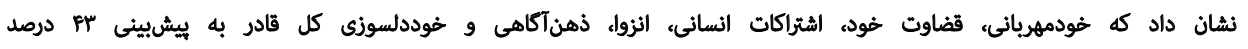

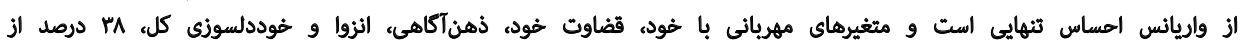

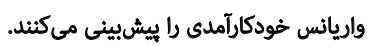

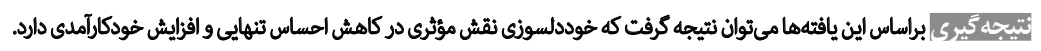

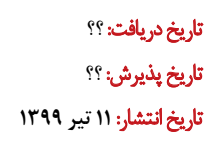

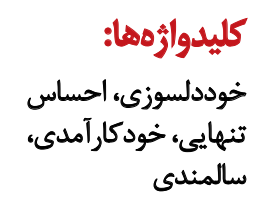

از تنهايى تعاريف مختلفى ارائه شده است. احساس تنهايى،

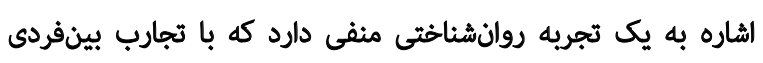

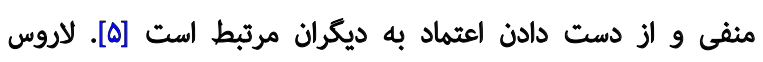

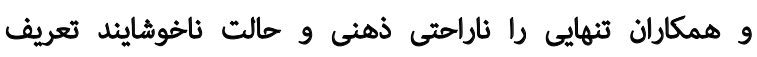

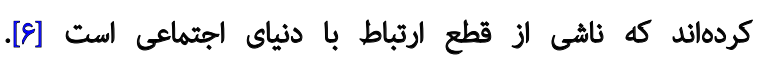

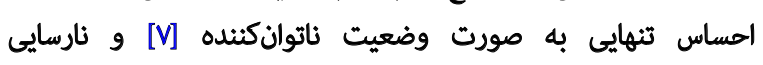

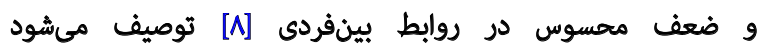

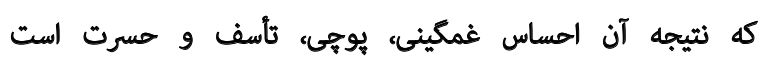

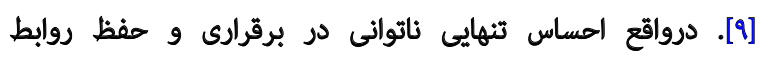

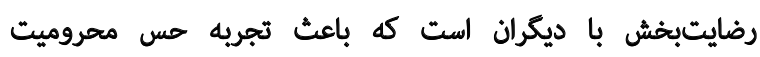

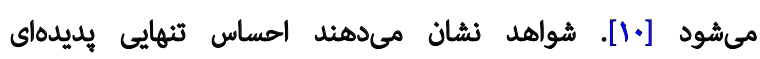

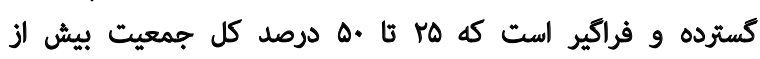

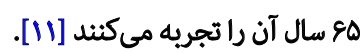
يكى از مؤلفههايى كه مىثواند نقش مؤثرى در كاهش احساس

سالمندى مرحلهاى از سير طبيعى زندكى انسان است كه براى

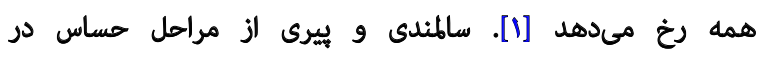

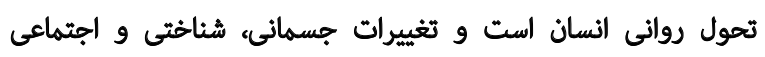

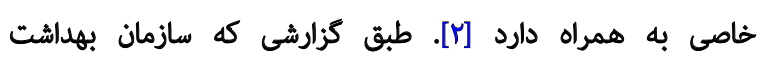

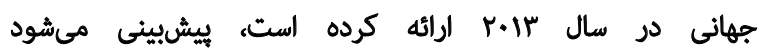

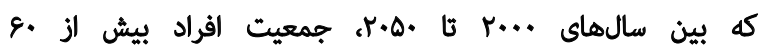

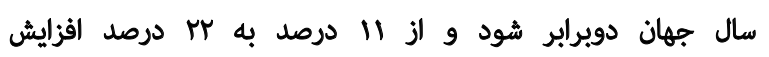

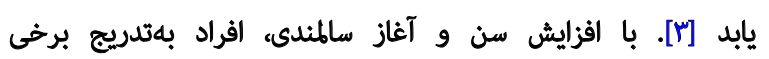

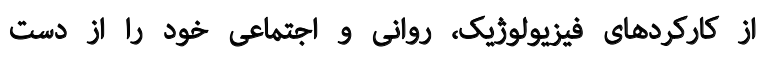

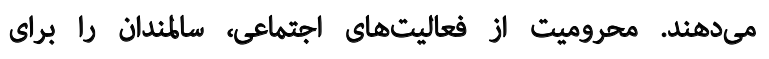

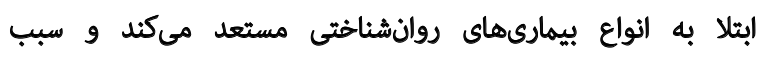

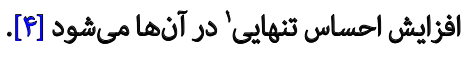

1. Loneliness

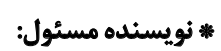

$$
\begin{aligned}
& \text { دكتر مهدى قزلسفلو } \\
& \text { نشانى: كنبد كاووس، دانشكاه كنبد كاووس، دانشكده علوم انسانى و تربيتى، كروه روانشناسى. }
\end{aligned}
$$

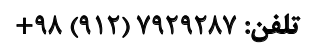

$$
\begin{aligned}
& \text { بست الكترونيكي: me.ghezelseflo@gmail.com }
\end{aligned}
$$


دوونى بيشتر و سطوح بالاترى الز هوش هيجانى، مهارتهاى

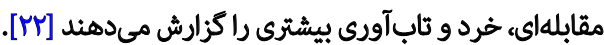

تحقيقات نشان دادهاند كه مهربانى با خود، الشتراكات انسانى و

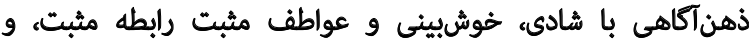

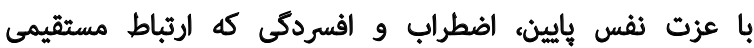

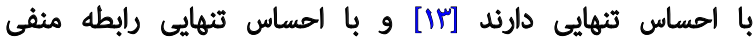

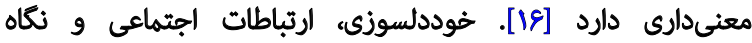

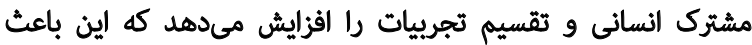

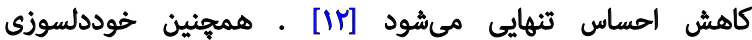
ارتباط منفى با اختلالات نوروتيك و سايكوتيك دارد كه هر دوى اين اختلالات به طور مثبت با الحساس ثنهايى رابطه مثبتى دارند

يزوهشها نشان ميدهد كه خوددلسوزي با بسيارى از سازههاي

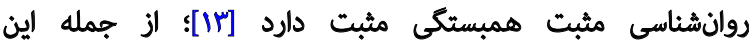

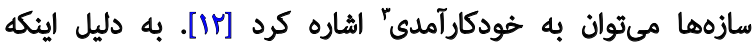
در فرايند سالمندى، سيستمهاى مختلف بدن تحليل مىرود و دران

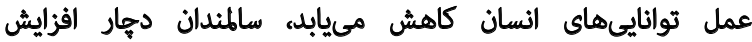
ناتوانى، كاهش اسثقلال و افزايش وابستكى به ديكائ ديكران و الحساس

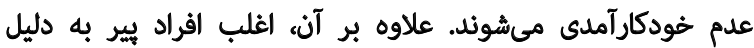

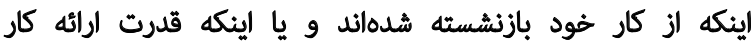

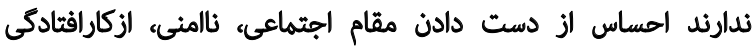

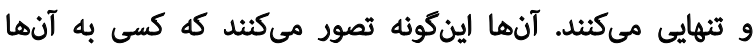

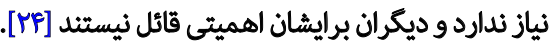

داويليس عنوان مىكند باورهاى خودكارآمدى شئاختهايى

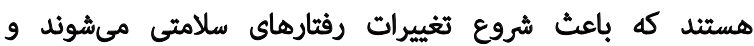

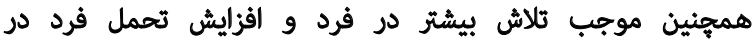
مقابل موانع و همهينين در مواقع شكست مىشوند [ها]]. والأه

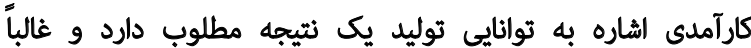

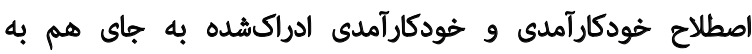

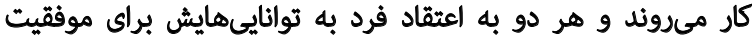

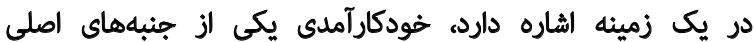

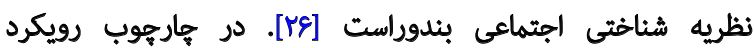

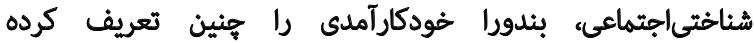

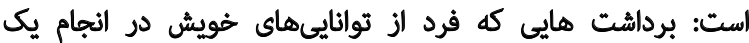
تكليف خاص دارد (به كونهاي كه تكليف موردنظر به صورت مفرد توايد

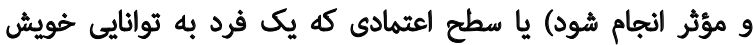

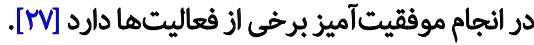

برخوردارى از احساس خودكارآمدى موجب ايجاد ارتباطهاى

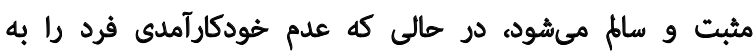

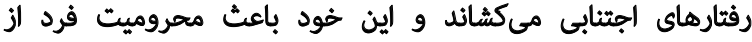

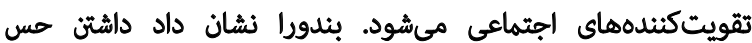

تنهايى سالمندان داشته باشد متغير خوددلسوزى" است. مفهوم

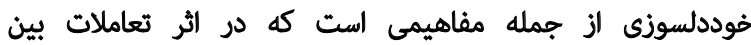

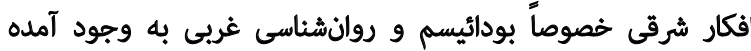

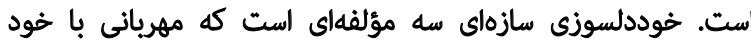

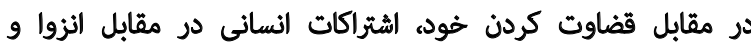

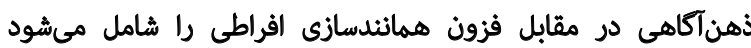

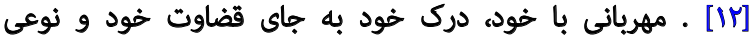

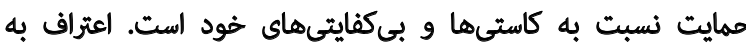

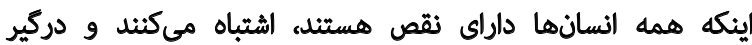

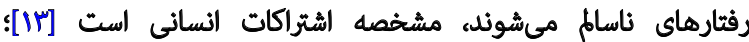

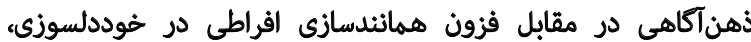

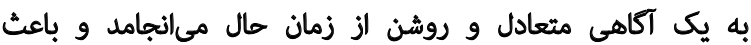
مىشود جنبههاي دردناك يك تجربه ثاديده كرفته نشود و و مكراً

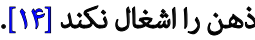

خوددلسوزى به عنوان كيفيت مواجه شدن با رنج و آسيب

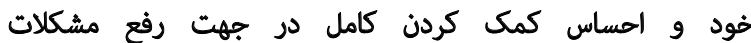

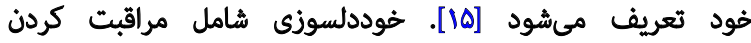

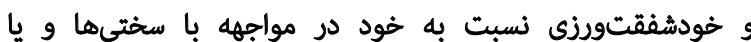

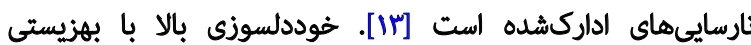
روانشناختى همراه الست و الز افراد در مقابل استرس حمايت

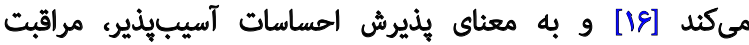
و مهربانى نسبت به خود، نكرش غيرارزيابانه نسبت به به نارسايىها و شكستهاى خويش و بازشناسى تجربيات فرد، تعريف مى شوده.

شواهد نشان ميدهد افرادى كه از خوديلسوزى بالآترى

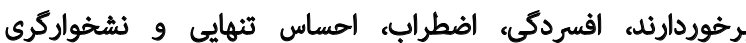

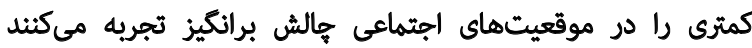

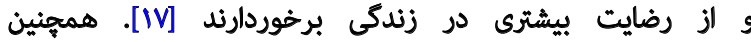

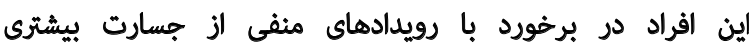

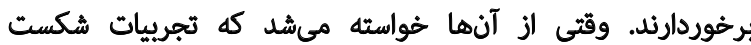

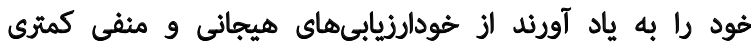

استفاده ميكردند [ili]

همئين مطالعاث نشان دادهاند كه افرادى كه خوددلسوزى

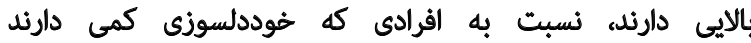

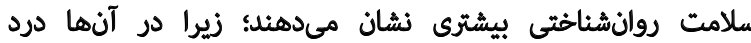

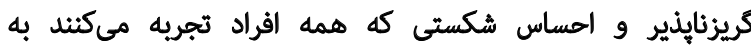

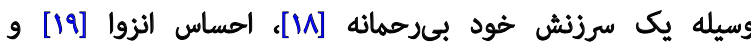

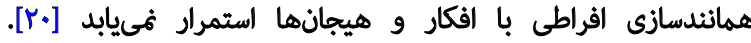

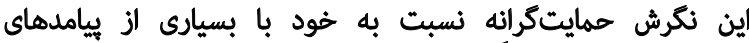

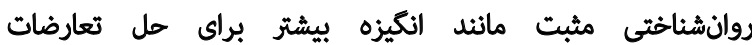

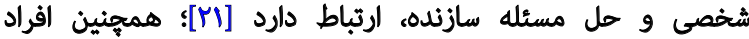

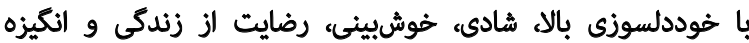




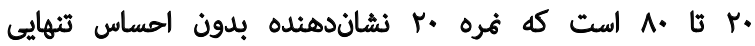

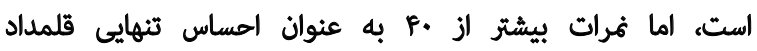

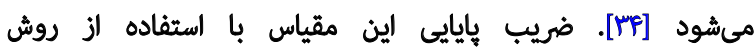

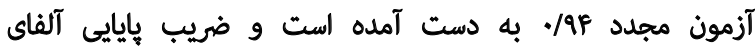

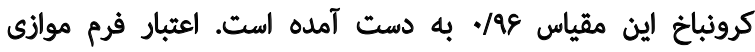

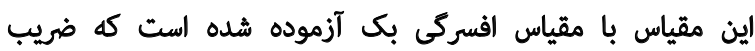

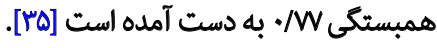

\section{مقياس خوددلسوزى (SCS)}

اين مقياس M Mؤال دارد كه توسط نف [ع/] براي سنجش

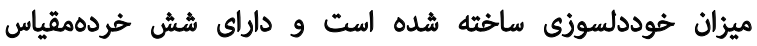

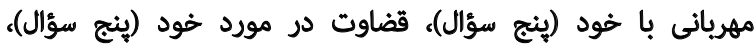

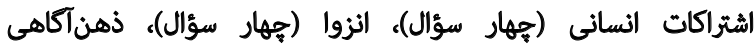

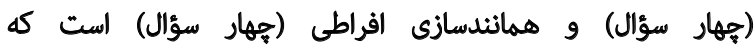

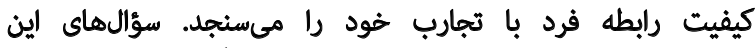

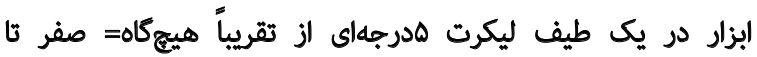

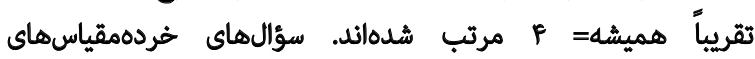

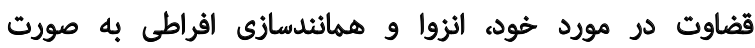

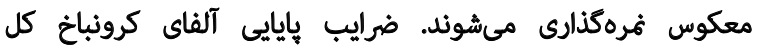

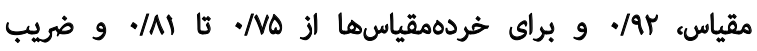

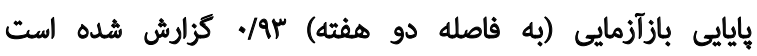

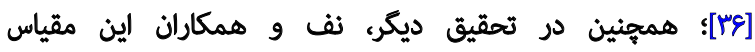

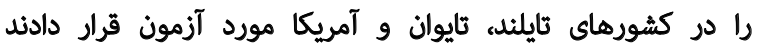

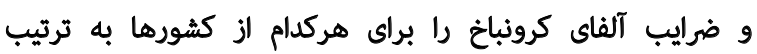

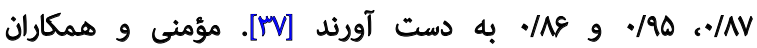

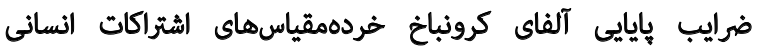

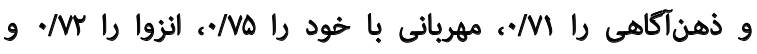

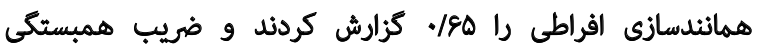

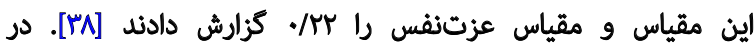

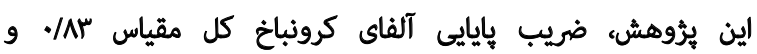

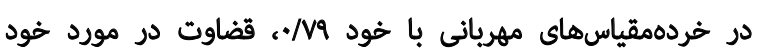

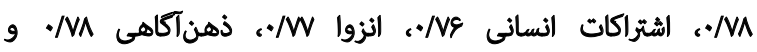

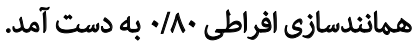

\section{يرسش نامه خودكار آمدى عمومي (GSE)}

اين مقياس توسط شرر و مادوكس" ساخته شده است و شامل

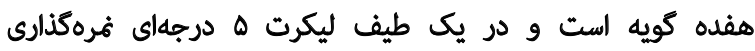

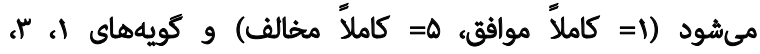

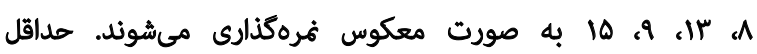

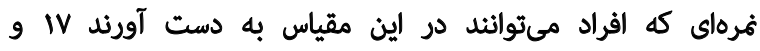

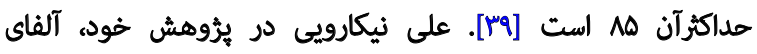

5. Self-compassion Scale

6. General Self-Efficacy Scale (GSE)

7. Sherer, Maddux
قوى خودكارآمدى، به سلامت روانشناختى افراد كمك ميكند

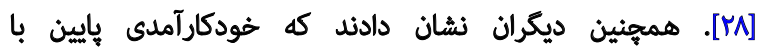

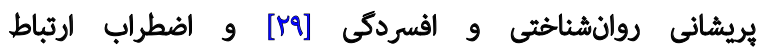

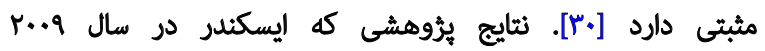

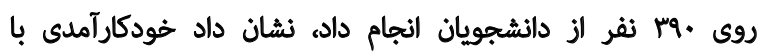

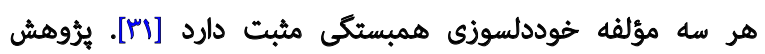

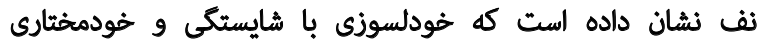
همبستكى مثبت معنادار دارد [IIT]

با توجه به اهميت دوران سالمندى و توجه كمتر به اين دوران

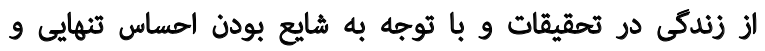

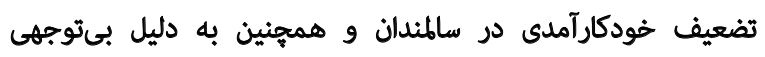

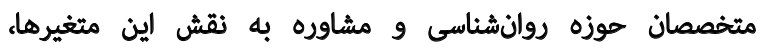

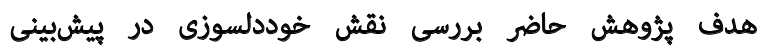

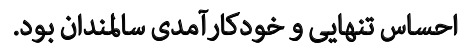

$$
\text { واش مطاله dاله }
$$

روش مطالعه حاضر توصيفى از نوع همبستكى بود. جامعه

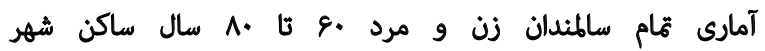

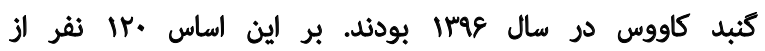

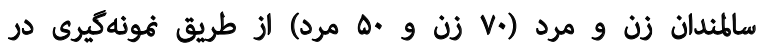

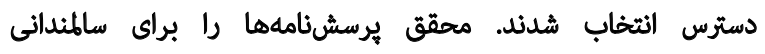

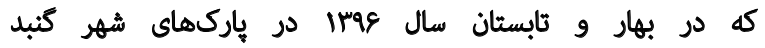

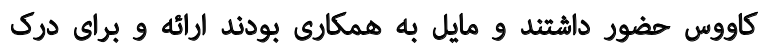

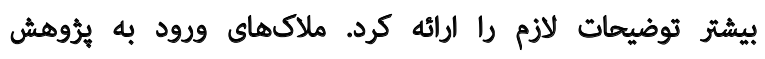

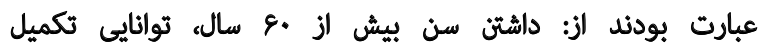

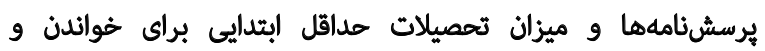

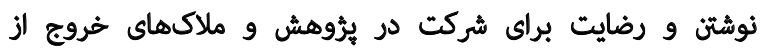

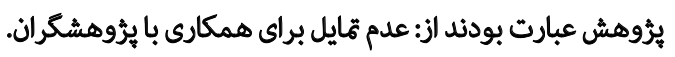

مقياس احساس ثنهاين (USLA)

راسل و همكارانش در سال IQVA براى ارزيابى تنهايى، نسخه اصلى مقياس احساس تنهايى بيستسؤالى را از دانشكاه كاليفرئيا،

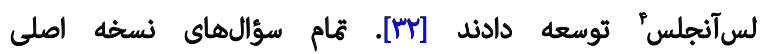
اين مقياس به صورت منفى بود. نسخه تجديدنظرشده (نسخه

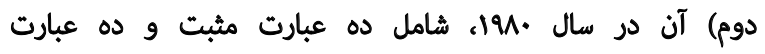

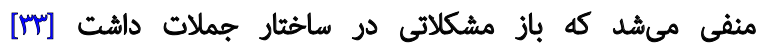

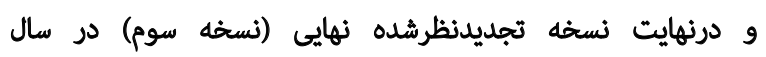

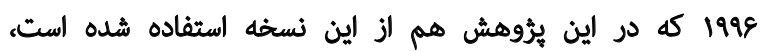

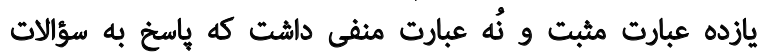

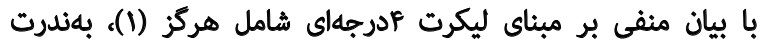

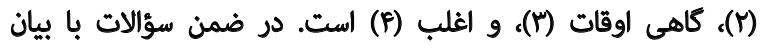

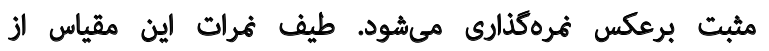

4. University of California, Los Angeles (UCLA) 
محرمانه خواهد بود. به منظور تجزيه و تحليل دادهها از شاخصهاى

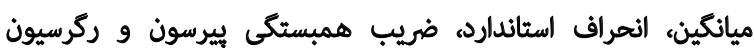

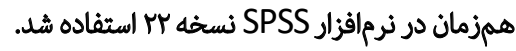

bergós

به منظور تجزيه و تحليل يافتهها، در يُروهش حاضر، دادهها در

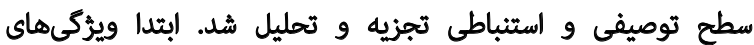

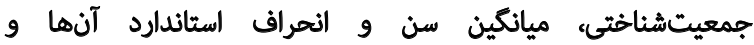

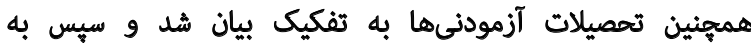

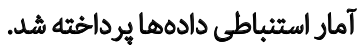

همان طور كه در جدول شماره ا مشاهده مىشوده ميانكين

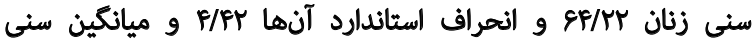

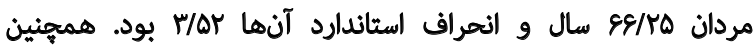

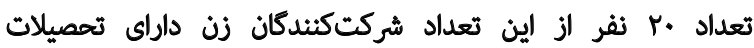

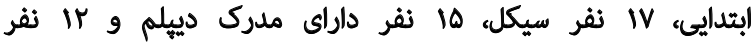

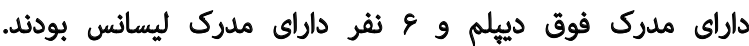

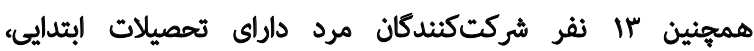

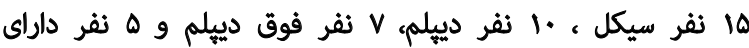

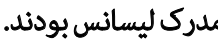

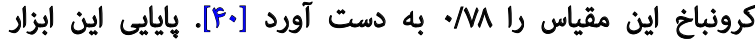

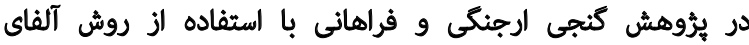

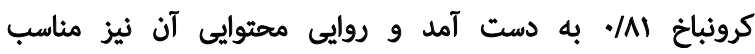

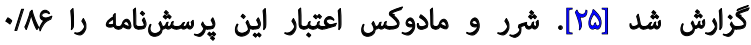

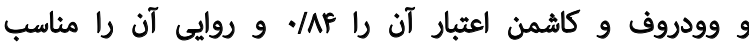

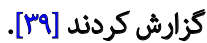

$$
\text { ورش الجرا }
$$

با توجه به هضور سالمندان در باركها در فصل بهار و تابستان

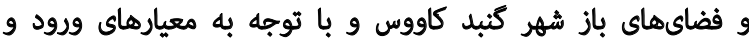

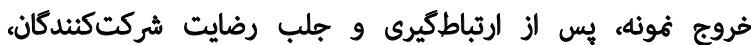

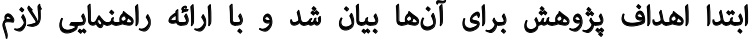

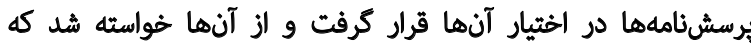

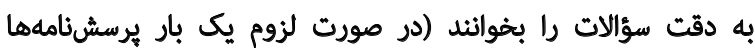

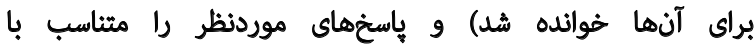

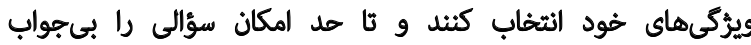

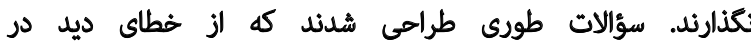

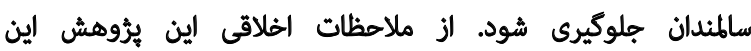

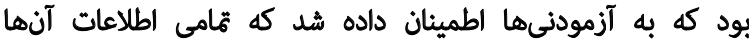

جدولا. توزيع فراوانى آزمودنى ها به تفكيك تحصيلات و ميانكين سن و انحراف استاندارد

\begin{tabular}{|c|c|c|c|c|c|c|c|}
\hline \multirow{2}{*}{ انحراف استاندارد } & \multirow{2}{*}{ ميانكين سن } & \multicolumn{5}{|c|}{ تحصيلات } & \multirow{2}{*}{ كروه } \\
\hline & & ليساتس & فوق دييلم & ييبلم & سيكل & ابتدايي & \\
\hline efer & $90 / \pi$ & 8 & ir & 10 & IV & r. & زنان \\
\hline r/AT & E\&/TA & $\Delta$ & 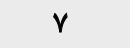 & 1. & 10 & ir & مردان \\
\hline
\end{tabular}

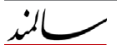

جدول r. ميانكين، انحراف استاندارد و ماتريس همبستكى بين متغيرها

\begin{tabular}{|c|c|c|c|c|c|c|c|c|c|}
\hline 9 & $\wedge$ & $v$ & 7 & - & $\varepsilon$ & $r$ & $r$ & 1 & متغيرها \\
\hline & & & & & & & & 1 & 1. احساس تنهايیى \\
\hline & & & & & & & 1 & $-.18 *^{*}$ & ז. مهربانى با خود \\
\hline & & & & & & 1 & $-\| / T^{*}$ & $.109^{\circ}$ & "ا. قضاوت خود \\
\hline & & & & & 1 &.$- / 8 Y^{*}$ & $.18 r^{\circ}$ & $-\cdot / \triangle A^{*}$ & f. اشتراكات أنسائى \\
\hline & & & & 1 & $-\Delta / \Delta \%$ & $.10 \mathrm{~A}^{*}$ & $-\cdot / e \theta^{*}$ & $.100^{\circ}$ & هـ اتزوا \\
\hline & & & 1 & $-\cdot /\left.\Delta\right|^{* *}$ & $\cdot(\Delta)^{*}$ & $-\cdot /\left.\Delta\right|^{*}$ & $.18 . *$ & $-.180^{*}$ & و ذهن أكاهى \\
\hline & & 1 & $-\cdot|\Delta|^{*}$ & $.109^{*}$ & $-* / \Delta *^{*}$ & $.18 T^{*}$ & $-* / P r *$ & $\cdot / \Delta r^{*}$ & V. فزون همانئنسازى \\
\hline & 1 & $-* \mid \Delta r^{*}$ & $.19 T^{*}$ & $-* /\left.\Delta\right|^{*}$ & $\cdot / \Delta \mathrm{r}^{*}$ & $-\mid / x^{*}$ &.$/ p r *$ & $-\cdot / \Delta \Delta^{*}$ & 1 خودكارآمدى \\
\hline 1 & . pre* & $-* / / q^{*}$ & $.10 F^{*}$ & $-* / 40^{*}$ & $. \mid \Delta)^{*}$ & $-* / \Gamma A^{*}$ & $\cdot \mid \Delta)^{*}$ &.$- / 4 T^{*}$ & 9.خوددلسوزي كل \\
\hline 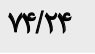 & $P V / M P$ & $\mid r / \Delta A$ & $I r / N$ & $I T / N$ & $11 / 9$. & $I r / r A$ & $11 / 91$ & $P T / 9 \Delta$ & ميانكين \\
\hline V/Tr & WIT & $r / m e$ & $f / F$. & $r / n$ & r/ar & $r / 99$ & $r / * \Delta$ & Ir/gr & أنحراف استاندارد \\
\hline
\end{tabular}


جدول r. خلاصه تحليل ركرسيون خطى ييشبينى احساس تنهايى بر اساس خوددلسوزى

\begin{tabular}{|c|c|c|c|c|c|c|c|}
\hline $\mathbf{P}$ & $\mathbf{F}$ & Adjusted R & $\mathbf{T}$ & B & $\boldsymbol{\beta}$ & متغير ييشبين & متغير ملاك \\
\hline $.1 .+4$ & & & $-r / 9 V$ & $-1 / 84$ &.$- / M T r$ & خودمهربانى & \\
\hline $.1 . .9$ & & & r/FA &.$/ 9 \Delta$ &.$|r A|$ & قضاوت خود & \\
\hline .1 .41 & & & $-Y / / r$ & $-.1 . \mu$ &.$- / T A F$ & اششراكات أسائى & \\
\hline $.1 . r A$ & $r / / r$ & Tr/ & $r / \pi r$ & $\cdot M$ & $\cdot / r \cdot r$ & انزوا & احساس تنهايى \\
\hline $1 . \pi r$ & & & $-M / T r$ & $-1 / 1 f$ &.$- / T \cdot \Gamma$ & ذهن آكاهى & \\
\hline $.1 . \mathrm{VA}$ & & & $I / N A$ & .199 &.$/ 109$ & فوزون همانثدسازى & \\
\hline $.1 . .9$ & & & $-Y / F A$ &.$- / 9 \Delta Y$ & $--1 \Delta \cdot 1$ & خوددلسوزى كل & \\
\hline
\end{tabular}

جدول \& . خلاصه تحليل ركرسيون خطى ييشبينى خودكارآمدى بر اساس خوددلسوزى

\begin{tabular}{|c|c|c|c|c|c|c|c|}
\hline $\mathbf{P}$ & $\mathbf{F}$ & Adjusted R & $\mathbf{T}$ & B & $\boldsymbol{\beta}$ & متغير ييشبين & متغير ملاك \\
\hline ./.1\% & & & r/AT & $1 / 199$ &.$/ N V$ & خودميهربانى & \\
\hline.$/ . r$ & & & $r / T \Delta$ & $-1 / 991$ &.$- / W E$ & قضاوت خود & \\
\hline ./ava & & &.$/ 41$ & .1 .5 & /..r & اثتراكات انسانى & خودكار آملعى \\
\hline r & $r \Delta / . \gamma$ & . & $-r / \Delta r$ & $-1 / 199$ &.$- / \pi r$ & انزوا & حودمارامدى \\
\hline .1 .1 & & & T/8Q & $V / A V$ &.$/ \pi f q$ & ذهن أكاهى & \\
\hline.$/ \Delta V$. & & & $-\cdot / \Delta V$ & $-+/ r 9 \Delta$ & $-.1 \cdot \Delta \Delta$ & فزون همانثدسازى & \\
\hline.$/ .+r$ & & & T/Ta & V/99 & .1994 & خوددلسوزى كل & \\
\hline
\end{tabular}

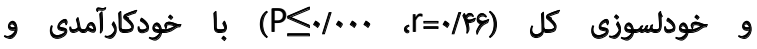

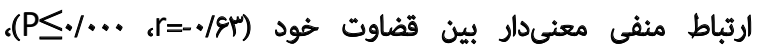

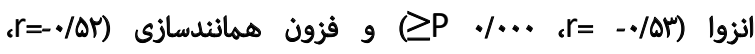

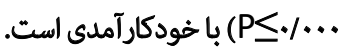

همانكونه كه در جدول شماره ب مشاهده مىشود، تركيب

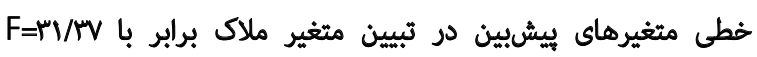

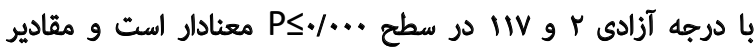

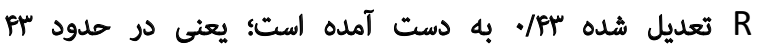

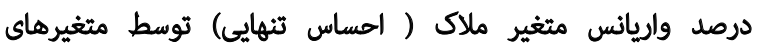

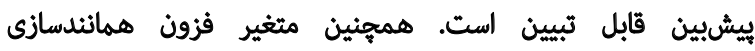
توان ييشبينى متغير ملاك (الحساس تثهايى) رانداشت.

همانكونه كه در جدول شماره م مشاهده مىشود، تركيب

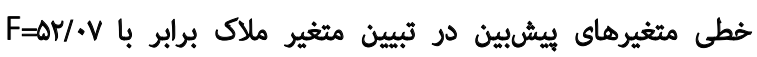

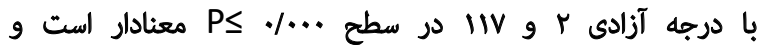

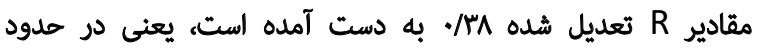

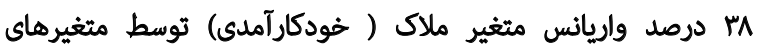

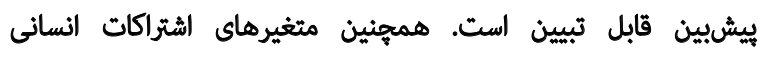
و فزون همانثدسازى توان ييشبينى متغير ملاك ( خودمكارآمدى) رانداشت. فزون.
مانطور كه در جدول شماره Y مشاهده مىشوده ميانكين و

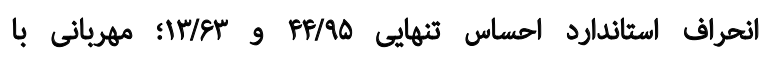

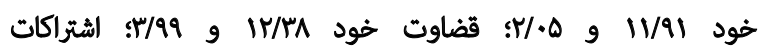

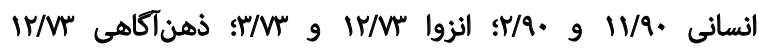

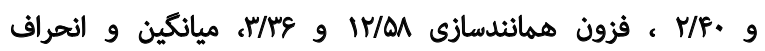

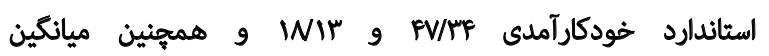

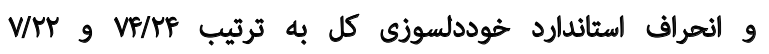

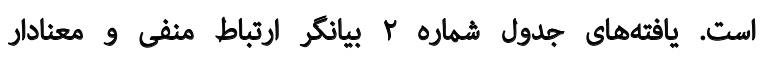

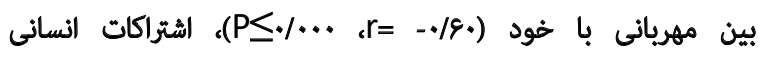

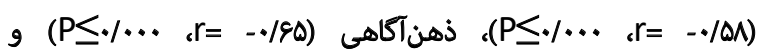

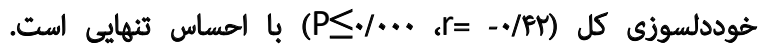

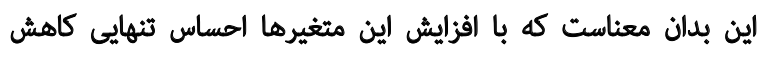

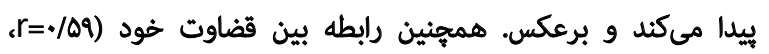
(PS

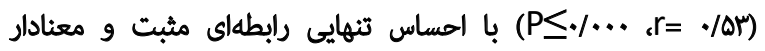

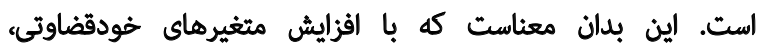

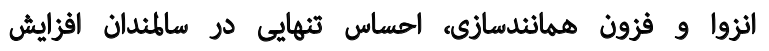

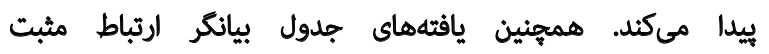

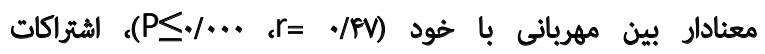
انسانى 
همجنين خوددلسوزى درواقع يك شكل سامل خوديذيرى

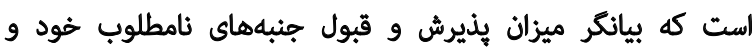

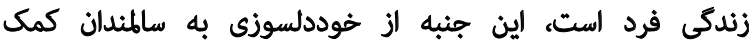

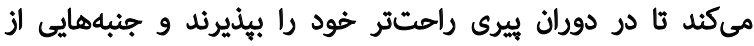

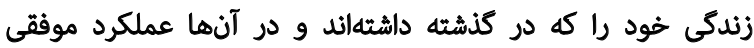

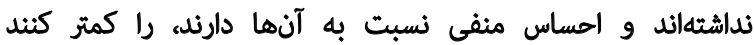

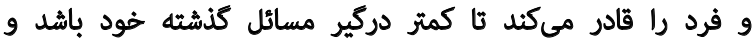

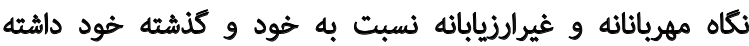

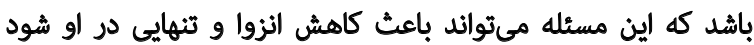

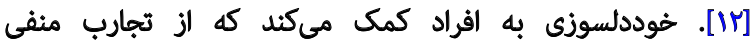

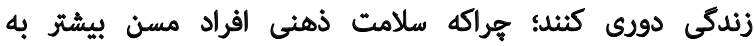

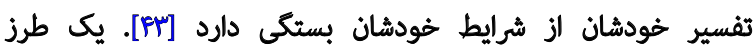

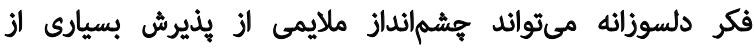

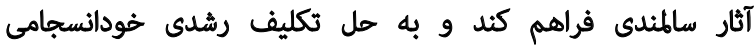

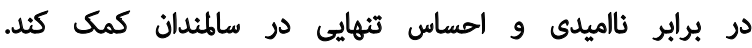

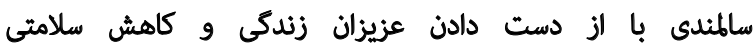

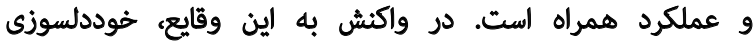

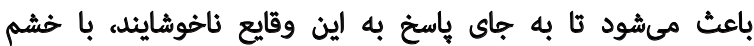

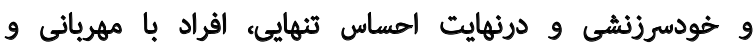

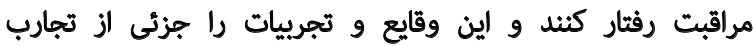

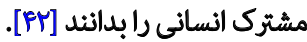

همجنين افراد با خوددلسوزى توائندىهاى روانشناختى

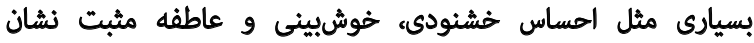

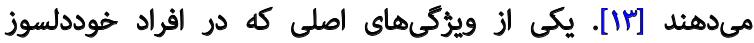

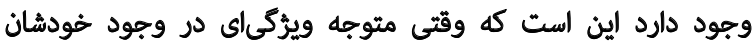
مىشوند كه آن را دوست ندارنده قضاوت سختكيرانه و و منتقدانه

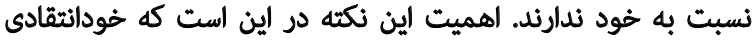

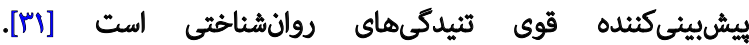

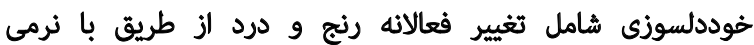

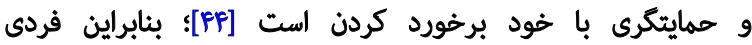

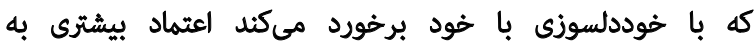

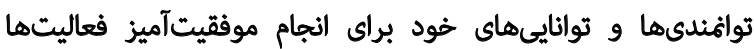

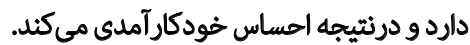

داورى افراد خوددلسوز در مورد خودشان در جهتى است

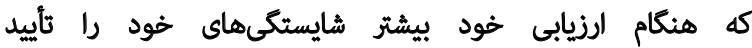

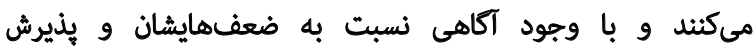

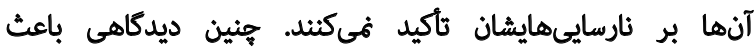

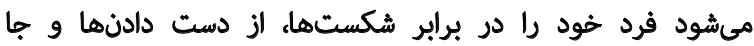

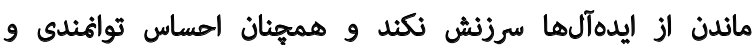

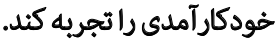

\section{تثيجليكيرىنهاييى}

با توجه به يافتهها مىتوان نتيجه كرفت كه خوددلسوزى بالا،
بحث

هدف يروهش حاضر بردسى نقش خوددلسوزى در بيشبينى

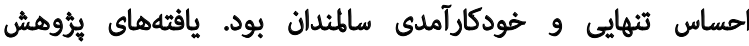

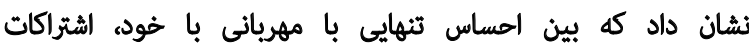

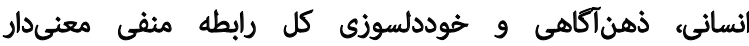

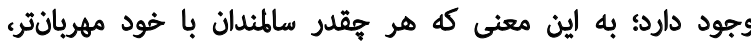

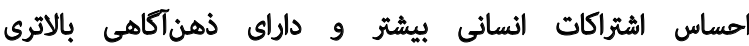

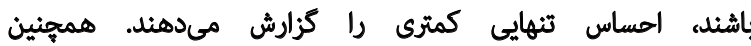

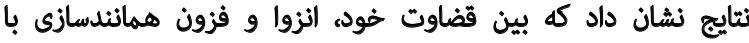

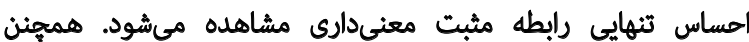

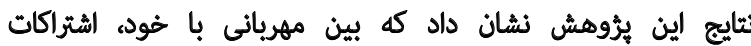

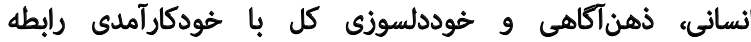

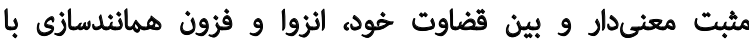

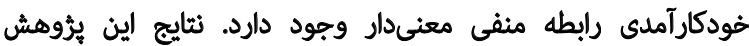

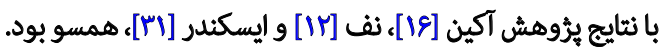

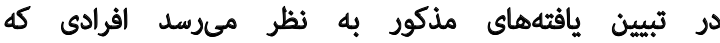

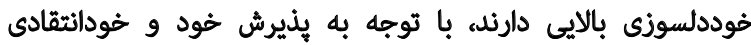

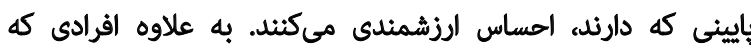

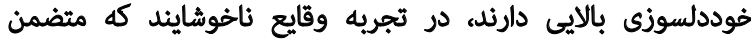

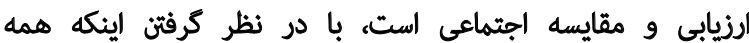

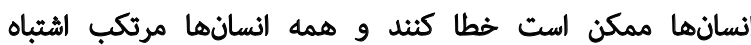

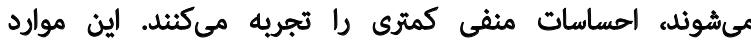

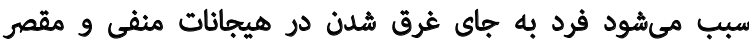

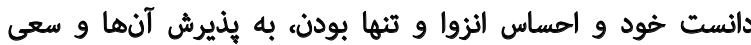

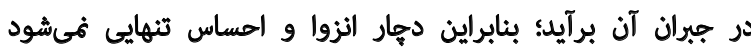

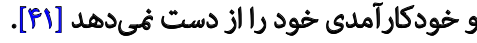

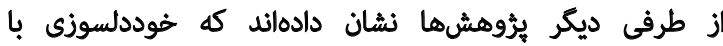

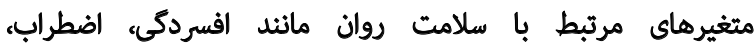

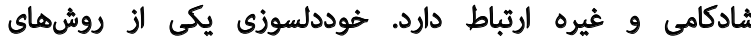

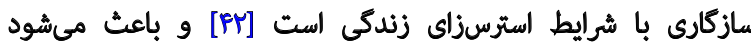

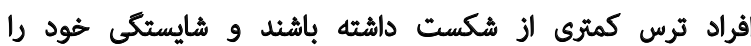

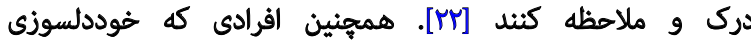

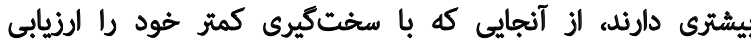

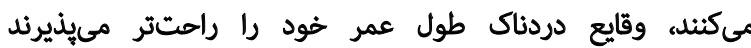

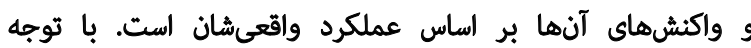
به اين يذيرش بالا در دوران سالمندى، اين الفراد كمتر دهار بهار

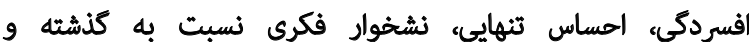

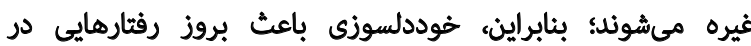

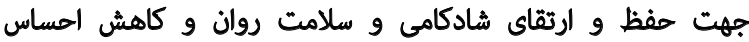

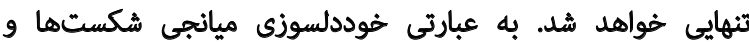

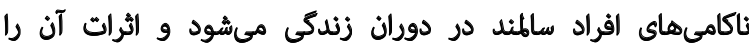

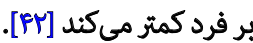


الحساس تنهايى را به طور منفى و خودكارآمدى را به طور مثبت

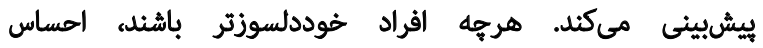

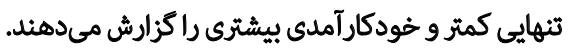

اكرجه نثايج اين مطالعه به طور بالقوه ميتواند مفيد باشده

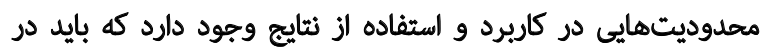

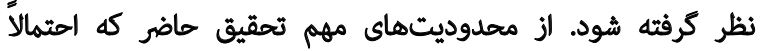

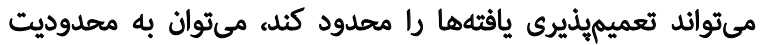

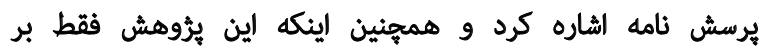

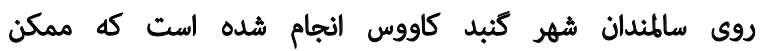

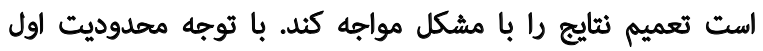

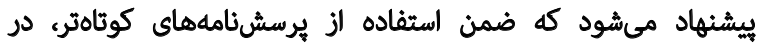

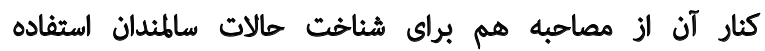

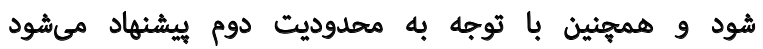
ئزوهشهاى مشابه در نقاط ديكر نيز النجام كيرد. ملاحظات اخالاقى

\section{ييروى از اصول اخلاق تيُوهش}

ثمامى اصول اخلاق در اين مقاله رعايت شده است . شركت

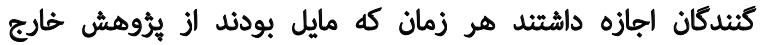

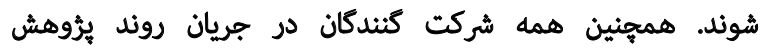

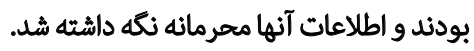

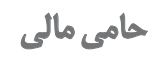

اين مماله حامى مالى ندارد.

$$
\text { مشاركت نويسندكان }
$$

مفهوم سازى، تحقيق و بررسى، منابع و نكارش بيش نويس، همه نويسندكان؛ روش شناسى، اعثبار سنجى، تحليل ، ويراستارى

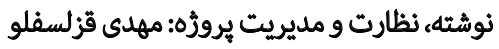

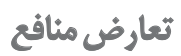

بنابر اظهار نظر نويسندكانء اين مقاله تعارض منافع ندارد 


\section{References}

[1] Sadri Damirchi E, Ghomi M, Esmaeli Ghazi Valoii F. [Effectiveness of life review group therapy on psychological well-being and the life expectancy of elderly women (Persian)]. Iranian Journal of Ageing. 2017; 12(3):312-25. [DOI:10.21859/sija.12.3.312]

[2] Zamani SN, Bahrainian SA, Ashrafi S, Moqtaderi Sh. [Effect of spiritual intelligence on quality of life and psychological well being among elderly living at nursing homes in Bandar Abbas (Persian)]. Journal of Geriatric Nursing. 2015; 1(4):82-94. http://jgn. medilam.ac.ir/article-1-136-fa.htm

[3] Group W. Study protocol for the World Health Organizationproject to develop a Quality of Life assessment instrument (WHOQOL). Quality of Life Research. 1993; 2(2):153-9. [DOI:10.1007/ BF00435734

[4] Sharifi M, Mohammad-Aminzadeh D, Soleimani Sefat E, Sudmand N, Younesi SJ. [Relationship of deterministic thinking with loneliness and depression in the elderly (Persian)]. Iranian Journal of Ageing. 2017; 12(3):276-87. [DOI:10.21859/sija.12.3.276]

[5] Rotenberg KJ, MacDonald KJ, King EV. The relationship between loneliness and interpersonal trust during middle childhood. The Journal of Genetic Psychology. 2004; 165(3):233-49. [DOI:10.3200/GNTP.165.3.233-249] [PMID]

[6] Larose S, Guay F, Boivin M. Attachment, social support, and loneliness in young adulthood: A test of two models. Personality and Social Psychology Bulletin. 2002; 28(5):684-93. [DOI:10.1177/0146167202288012]

[7] VanderWeele TJ, Hawkley LC, Thisted RA, Cacioppo JT. A marginal structural model analysis for loneliness: Implications for intervention trials and clinical practice. Journal of Consulting and Clinical Psychology. 2011; 79(2):225. [DOI:10.1037/a0022610] [PMID] [PMCID]-35

[8] Parkhurst JT, Hopmeyer A. Developmental change in thesources of loneliness in childhood and adolescence: Constructing a theoretical model. Loneliness in Childhood and Adolescence. 1999:56-79. [DOI:10.1017/CBO9780511551888.004]

[9] Asher SR, Paquette JA. Loneliness and peer relations in childhood. Current Directions in Psychological Science. 2003; 12(3):75-8. [DOI:10.1111/1467-8721.01233]

[10] Heinrich LM, Gullone E. The clinical significance of loneliness: A literature review. Clinical Psychology Review. 2006; 26(6):695-718. [DOI:10.1016/j.cpr.2006.04.002] [PMID]

[11] Chiang KJ, Chu H, Chang HJ, Chung MH, Chen $\mathrm{CH}$, Chiou $\mathrm{HY}$,et al. The effects of reminiscence therapy on psychological well-being, depression, and loneliness among the institutionalized aged. International Journal of Geriatric Psychiatry. 2010; 25(4):380-8. [DOI:10.1002/gps.2350] [PMID]

[12] Neff K. Self-compassion: An alternative conceptualization of a healthy attitude toward oneself. Self and identity. 2003; 2(2):85-101. [DOI:10.1080/15298860309032]

[13] Neff KD, Kirkpatrick KL, Rude SS. Self-compassion and adaptive psychological functioning. Journal of research in personality. 2007; 41(1):139-54. [DOI:10.1016/j.jrp.2006.03.004]

[14] Neff KD. The role of self-compassion in development: A healthier way to relate to oneself. Human Development. 2009; 52(4):211-4. [DOI:10.1159/000215071] [PMID] [PMCID]
[15] Wren AA, Somers TJ, Wright MA, Goetz MC, Leary MR, Fras $\mathrm{AM}$, et al. Self-compassion in patients with persistent musculoskeletal pain: Relationship of self-compassion to adjustment to persistent pain. Journal of Pain and Symptom Management. 2012; 43(4):759-70. [DOI:10.1016/i.jpainsymman.2011.04.014] [PMID]

[16] Akin A. Self-compassion and Loneliness. International Online Journal of Educational Sciences. 2010; 2(3)702-18. http://citeseerx.ist.psu.edu/viewdoc/summary?doi=10.1.1.422.382

[17] Raes F. Rumination and worry as mediators of the relationship between self-compassion and depression and anxiety. Personality and Individual Differences. 2010; 48(6):757-61. [DOI:10.1016/j. paid.2010.01.023]

[18] Blatt SJ, Quinlan DM, Chevron ES, McDonald C, Zuroff D. Dependency and self-criticism: Psychological dimensions of depression. Journal of Consulting and Clinical Psychology. 1982; 50(1):113-24. [DOI:10.1037/0022-006X.50.1.113] [PMID]

[19] Wood JV, Saltzberg JA, Neale JM, Stone AA, Rachmiel TB. Self-focused attention, coping responses, and distressed mood in everyday life. Journal of Personality and Social Psychology. 1990; 58(6):1027-36. [DOI:10.1037/0022-3514.58.6.1027] [PMID]

[20] Nolen-Hoeksema S. The role of rumination in depressive disorders and mixed anxiety/depressive symptoms. Journal of Abnormal Psychology. 2000; 109(3):504-11. [DOI:10.1037/0021843X.109.3.504] [PMID]

[21] Baker LR, McNulty JK. Self-compassion and relationship maintenance: The moderating roles of conscientiousness and gender. Journal of Personality and Social Psychology. 2011 100(5):853-73. [DOI:10.1037/a0021884] [PMID] [PMCID]

[22] Neff KD, Hsieh YP, Dejitterat K. Self-compassion, achievement goals, and coping with academic failure. Self and Identity. 2005; 4(3):263-87. [DOI:10.1080/13576500444000317]

[23] Cheng H, Furnham A. Personality, peer relations, and selfconfidence as predictors of happiness and loneliness. Journal of Adolescence. 2002; 25(3):327-39. [DOI:10.1006/jado.2002.0475] [PMID]

[24] Melzer D, McWilliams B, Brayne C, Johnson T, Bond J. Profile of disability in elderly people: Estimates from a longitudinal population study. The BMJ. 1999; 318(7191):1108-11. [DOI:10.1136/ bmj.318.7191.1108] [PMID] [PMCID]

[25] Gangi Arjangi M, Farahani MN. [The relationship between job stress and self efficacy with life satisfaction in gas accident workers from Isfahan gas company (Persian)]. Journal of Research in Psychological Health. 2009; 3(7):15-24. https:/ /b2n.ir/306627

[26] St Charles L. Mindfulness, self-compassion, self-efficacy, and locus of control: Examining relationships between four distinct but theoretically related concepts [MSc. thesis]. Oregon: Pacific University; 2010. https://core.ac.uk/download/pdf/48847255. pdf

[27] Gan Z, Liu F, Yang CC. Student-teachers' self-efficacy for instructing self-regulated learning in the classroom. Journal of Education for Teaching. 2020; 46(1):120-3. https://doi.org/10.1 080/02607476.2019.1708632

[28] Bandura, A. Adolescent development from an agentic perspective. In: F. Pajares \& T. Urdan, edirors. Self-efficacy beliefs of adolescents. Charlotte: Information Age Publishing; 2006. 
[29] Chen J, Liu TQ, Zheng MJ, Chen C. Relationships between self-esteem, self-efficacy, self-concept, and depression. Chinese Journal of Clinical Psychology. 2010; 18(6):799-801. http:/ /caod. oriprobe.com/articles/25848960/Relationship_Between_Self_ esteem_Self_efficacy_Self_concept_and_Depres.htm

[30] Endler NS, Speer RL, Johnson JM, Flett GL. General selfefficacy and control in relation to anxiety and cognitive performance. Current Psychology. 2001; 20(1):36-52. [DOI:10.1007/ s12144-001-1002-7]

[31] Iskender M. The relationship between self-compassion, selfefficacy, and control belief about learning in Turkish university students. Social Behavior and Personality: An International Journal. 2009; 37(5):711-20. [DOI:10.2224/sbp.2009.37.5.711]

[32] Russell D, Peplau LA, Ferguson ML. Developing a measure of loneliness. Journal of Personality Assessment. 1978; 42(3):290-4. [DOI:10.1207/s15327752jpa4203_11] [PMID]

[33] Peplau LA, Cutrona CE. The revised UCLA Loneliness Scale: Concurrent and discriminant validity evidence. Journal of Personality and Social Psychology. 1980; 39(3):472-80. [DOI:10.1037/0022-3514.39.3.472]

[34] Russell DW. UCLA loneliness scale (Version 3): Reliability, validity, and factor structure. Journal of Personality Assessment. 1996; 66(1):20-40. [DOI:10.1207/s15327752jpa6601_2] [PMID]

[35] Daniel K. Loneliness and depression among university Studentsin Kenya. Global Journal of Human Social Science Arts \& Humanities. 2013; 13(4):11-8. https:/ /globaljournals.org/GJHSS Volume13/2-Loneliness-and-Depression-among-University.pdf

[36] Neff K. The development and validation of a scale to measure self-compassion. Self and Identity. 2003; 2(3):223-50. [DOI:10.1080/15298860309027]

[37] Neff KD, Pisitsungkagarn K, Hsieh YP. Self-compassion and self-construal in the United States, Thailand, and Taiwan. Journal of Cross-Cultural Psychology. 2008; 39(3):267-85. [DOI:10.1177/0022022108314544]

[38] Momeni F, Shahidi S, Mootabi F, Heydari H. [Psychometric properties of a Farsi version of the Self-Compassion Scale (Persian)]. Contemporary Psychology. 2014; 8(2):27-40. https://b2n. ir/361195

[39] Sherer M, Maddux JE, Mercandante B, Prentice-Dunn S, Jacobs B, Rogers RW. The self-efficacy scale: Construction and validation. Psychological Reports. 1982; 51(2):663-71. [DOI:10.2466/ pr0.1982.51.2.663]

[40] Alinikavari R. [Relationship between self-efficacy and mental health of secondary school student in babol in the academic years 2001 (Persian)] [MA. thesis]. Tehran: Allameh Tabatabei University; 2002.

[41] Neff KD. Self-compassion, self-esteem, and well-being. Social and Personality Psychology Compass. 2011; 5(1):1-2. [DOI:10.1111/j.1751-9004.2010.00330.x]

[42] Neff KD, McGehee P. Self-compassion and psychological resilience among adolescents and young adults. Self and Identity. 2010; 9(3):225-40. [DOI:10.1080/15298860902979307]

[43] Siedlecki KL, Tucker-Drob EM, Oishi S, Salthouse TA. Life satisfaction across adulthood: Different determinants at different ages? The Journal of Positive Psychology. 2008; 3(3):153-64. [DOI:10.1080/17439760701834602] [PMID] [PMCID]
[44] Newby K. The Effects of Yoga with Meditation (YWM) on Self-Criticism, Self-Compassion, and Mindfulness [PhD. thesis]. Philadelphia: College of Osteopathic Medicine; 2014. https:// digitalcommons.pcom.edu/cgi/viewcontent.cgi?article=1303\&c ontext=psychology_dissertations 